\title{
89 Van der Hoeve de Kleyn syndrome
}

A Microscopic fractures with subsequent healing is the probable cause of stapedo-vestibular fixation.

B Deafness does not occur without evidence of fractures.

C Compliance values are low.

D Stapedectomy is usually deferred until several years after spontaneous fractures have ceased.

E Amelogenesis imperfecta may be associated.

\section{In late syphilis in the temporal bone}

A Cochlear duct hydrops is a very common feature.

$B$ Hennebert's sign may be positive.

C Steroids may improve the hearing deficit.

D Interstitial keratitis may have occurred several years previously.

E Tullio phenomena may be a symptom.

\section{Congenital deafness}

A Rubella is an infrequent prenatal cause of sensorineural deafness.

B A white forelock and heterochromia iridium may be associated.

C Screening tests can only be reliably performed after 12 months of age.

D A high tone loss is characteristic of cases caused by rhesus incompatibility.

E Electric response audiometry is not particularly useful in assessing auditory function. 